\title{
Application of Athermal Recombination Corrected DPA Model in Nuclear Data Processing Code NECP-Atlas
}

\author{
Wen Yin, Tiejun $\mathrm{Zu}^{*}$, and Liangzhi Cao \\ Xi'an Jiaotong University \\ No.28, Xianning West Road, Xi'an, Shaanxi, China \\ yinwen@stu.xjtu.edu.cn, tiejun@xjtu.edu.cn,caolz@mail.xjtu.edu.cn
}

\begin{abstract}
The Displacement per Atom (DPA) is an important factor used to quantify the irradiation damage of materials. The radiation damage energy production cross section obtained by nuclear data processing code is an essential nuclear data used to calculate the DPA of materials. Nuclear data processing code, such as NJOY, adopts NRT-DPA model to calculate the radiation damage energy production cross section. However, the NRT-DPA model has several well-known limitation. Especially, the number of radiation defects produced in energetic cascades in metals is only $\sim 1 / 3$ the NRT-DPA model prediction. To improve the precision accuracy of DPA calculation, the state-of-the-art Athermal Recombination Corrected DPA (ARC-DPA) model is adopted in heat production and radiation damage energy production cross section module of nuclear data processing code NECP-Atlas. ARCDPA model can be obtained by simply multiplying with the new efficiency function. The parameters in the new efficiency function are material constants that can be determined for a given material from Molecular Dynamics (MD) simulations or experiments. The material constants of some materials are obtained by MD simulations firstly in this paper. Numerical results show that the radiation damage cross section produced by nuclear data processing code NECP-Atlas adopting NRT-DPA model can agree well with the NJOY code. What's more, the results from NECP-Atlas adopting ARC-DPA model can provide more physically realistic descriptions of primary defect and agree well with the results from MD simulation numerically.
\end{abstract}

KEYWORDS: DPA; radiation damage energy production cross section; ARC-DPA model; NECP-Atlas; molecular dynamics simulation

\section{INTRODUCTION}

It is well known that the materials of nuclear systems undergo detrimental changes in their physical and mechanical properties due to exposure to high levels of incident radiations, such as neutrons, gammas and charged particles. DPA is a very important factor used to quantify the effect of incident radiations. The calculation of DPA can use transport code and the nuclear data library of transport code is generated by nuclear data processing code. Traditional nuclear data processing code, such as NJOY, adopts NRT-DPA model to calculate the radiation damage energy production cross section used by transport code to calculate DPA of materials. Kinchin and Pease proposed the KP-DPA model in 1955 [1]. The NRT-DPA model is improved from KP-DPA model in 1975 [2]. With the improvement of computational ability and the process of MD simulation, it is found that the current international standard for quantifying this

\footnotetext{
${ }^{*}$ Corresponding author. Tel: +86 298266 8916; fax: +86 2982668916
} 
energetic particle damage, the NRT-DPA model, has nowadays several well-known limitations. In particular, the number of radiation defects produced in energetic cascades in metals is only $\sim 1 / 3$ the NRTDPA model prediction [3]. Recently, an improved model named ARC-DPA model is proposed by Nordlund [4], which can provide more physically realistic descriptions of primary defect creation in materials. ARC-DPA model can be extended from the NRT-DPA model by multiplying the new efficiency function. The parameters in the new efficiency function are material constants that can be determined for a given material from Molecular Dynamics (MD) simulations or experiments. However, the material constants are given only for several materials in Ref. 4. Though Konobeyev [5] gives material constants for 81 materials by deriving the systematics based on their correlations with various material properties from the observations made in extensive MD simulations and experiments, the DPA results using material constants provided by Konobeyev can't be consistent with that using MD simulations. In order to realize the more physically realistic DPA calculation, the ARC-DPA model is used in the nuclear data processing code NECP-Atlas. And the material constants of some materials are obtained by MD simulations in this paper.

This paper is organized as follows. The methodologies used in the present work are described in Section 2. In Section 3, some numerical results and discussion are presented. Some conclusions are given in Section 4.

\section{METHODS}

Quantification of the amount of displacement damage introduced by energetic particle interactions in matter is important for a broad range of fundamental science and applied engineering applications. Kinchin and Pease developed the basis for an early model to calculate DPA by considering kinetic energy transfers above a threshold material-specific displacement energy in 1955 [1]. The current international standard is the Norgett-Robinson-Torrens (NRT)-DPA model [2].

\subsection{NRT-DPA model}

The NRT-DPA model is improved from Kinchin-Pease (KP) model and it can be given by:

$$
N\left(E_{a}\right)=\left\{\begin{array}{lr}
0, & 0<E_{a}<E_{d} \\
1, & E_{d}<E_{a}<\frac{2 E_{d}}{0.8} \\
\frac{0.8 E_{a}}{2 E_{d}}, & \frac{2 E_{d}}{0.8}<E_{a}<\infty
\end{array}\right.
$$

where $E_{a}$ is the Lindhard's damage energy [6]; $E_{d}$ is the threshold energy of atomic displacement, and the constant coefficient 0.8 is deduced from the Binary Collision Approximation (BCA) calculations [7]. The damage energy is computed with the PKA kinetic energy $E_{P K A}$ by $E_{a}=E_{P K A} \times P\left(E_{P K A}\right)$, where $P$ is the partition function that measures the fraction of $E_{P K A}$ left in atomic motion and $1-P$ represents the fraction of energy lost to electronic excitation [8]. The Robinson partition function is used in NRT-DPA model and can be written as:

$$
P\left(E_{P K A}\right)=\frac{1}{1+F_{L}\left(3.4008 \varepsilon^{1 / 6}+0.40244 \varepsilon^{3 / 4}+\varepsilon\right)}
$$


where $F_{L}=0.133745 Z^{2 / 3} A^{-1 / 2}, \varepsilon=E_{P K A} / E_{L}$ with $E_{L}=86.931 Z^{7 / 3}, \mathrm{Z}$ and $\mathrm{A}$ are the atomic number and the atomic mass number, respectively.

\subsection{ARC-DPA model}

It can be concluded from MD simulations and experimental results that the number of defects that really survive in the irradiated materials is much lower than what is predicted by the NRT model. The MD simulations of displacement damage cascades in metals have consistently shown closeness to the experimentally observed damage recombination. The results in Ref. 4 suggest that the defect production efficiency $\xi$, which is the ratio of the true number of existing defects to that predicted by NRT model, is much less than 1 for recoil energies above $\sim 1 \mathrm{keV}$. Thus, the ARC-DPA model is improved from NRTDPA model and it can be given by:

$$
N\left(E_{a}\right)=\left\{\begin{array}{cc}
0, & 0<E_{a}<E_{d} \\
1, & E_{d}<E_{a}<\frac{2 E_{d}}{0.8} \\
\frac{0.8 E_{a}}{2 E_{d}} \xi\left(E_{a}\right), & \frac{2 E_{d}}{0.8}<E_{a}<\infty
\end{array},\right.
$$

with the new efficiency function $\xi\left(E_{a}\right)$ given by:

$$
\xi\left(E_{a}\right)=\frac{1-c}{\left(2 E_{d} / 0.8\right)^{b}} E_{a}^{b}+c \xi\left(E_{a}\right)=\frac{1-c}{\left(2 E_{d} / 0.8\right)^{b}} E_{a}^{b}+c
$$

where $b$ and $c$ are material constants, that need to be determined for a given material from MD simulations or experiments. It is easy to modify computer codes that now use the NRT-DPA model by simply multiplying with the function $\xi\left(E_{a}\right)$.

\subsection{Material constant fitting}

Material constants, $\mathrm{b}$ and $\mathrm{c}$, are important parameter for the application of ARC-DPA model. Only several types of material give material constants when the ARC-DPA model is proposed. Though material constants are estimated for 70 materials from Li to $\mathrm{U}$ using available experimental data by Konobeyev [5], it also need to pay attention to accuracy. Thus, the material constants of some materials are obtained by MD simulations firstly in this paper, including $\mathrm{Zr}$, Ti and Al.

The figure of the number of Frenkel pairs versus the kinetic energy of the primary knock-on atom (PKA) is shown in Fig. 1. These data obtained by MD simulation is got from Ref. 9-10.

It can be concluded that the number of Frenkel pairs is a function of damage energy $E_{a}$. Because the Fig. 1 give the data of the number of Frenkel pairs versus the kinetic energy of the PKA, it need to change the $E_{P K A}$ into $E_{a}$ by multiplying the partition function shown in Eq. (2). The least square method is adopted for material constants fitting. The fitting results are shown in Table I. 


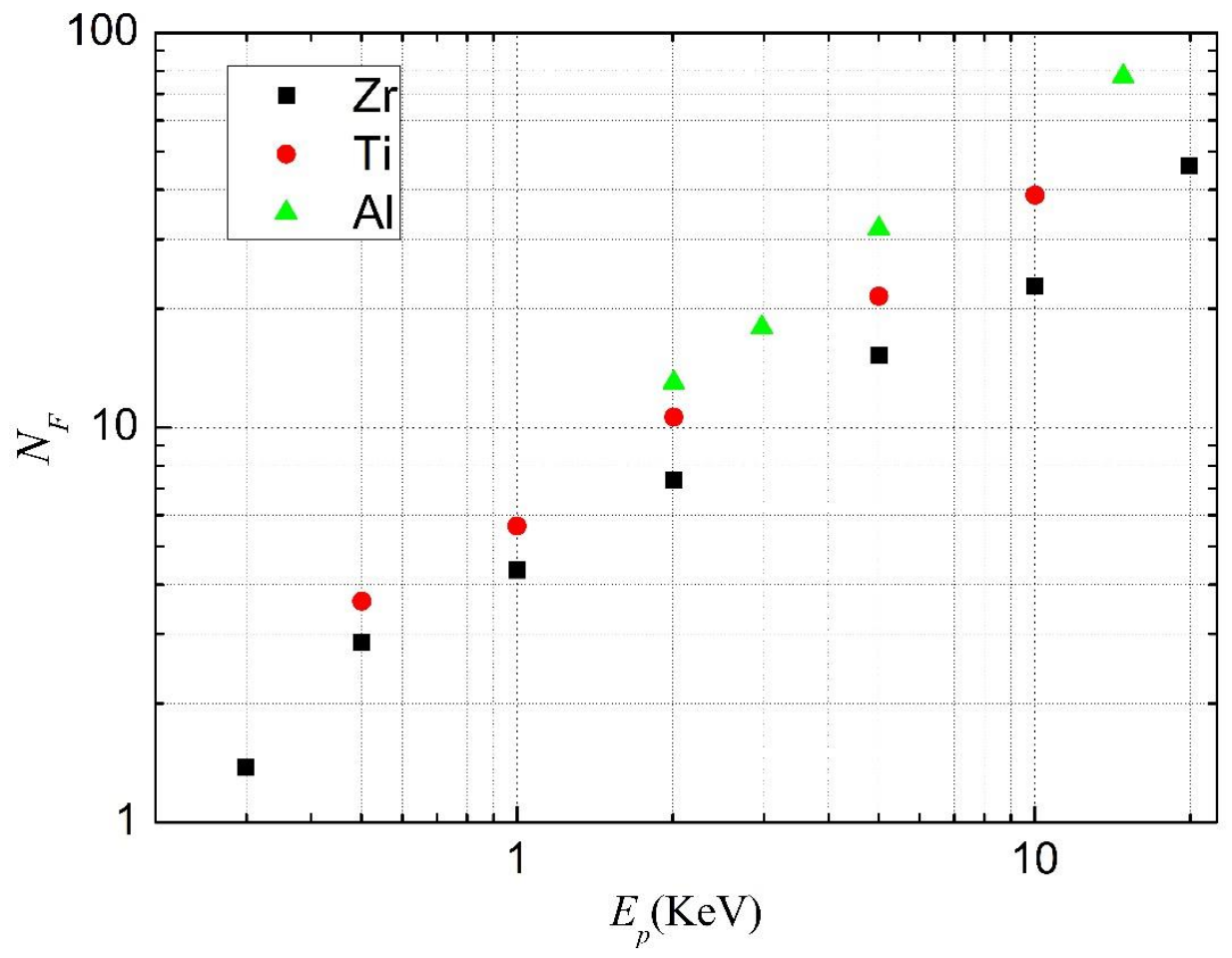

Figure 1. $\log -\log$ plots of $N_{F}$ vs $E_{p}$ for $\mathrm{Zr}$, Ti and Al

Table I. Material constants

\begin{tabular}{|c|c|c|c|c|}
\hline Material & $E_{d}(\mathrm{eV})$ & $b$ & $c$ & Reference \\
\hline $\mathrm{Fe}$ & 40 & -0.568 & 0.286 & {$[4]$} \\
\hline $\mathrm{Cu}$ & 33 & -0.68 & 0.16 & {$[4]$} \\
\hline $\mathrm{Ni}$ & 39 & -1.01 & 0.23 & {$[4]$} \\
\hline $\mathrm{Pd}$ & 41 & -0.88 & 0.15 & {$[4]$} \\
\hline $\mathrm{Pt}$ & 42 & -1.12 & 0.11 & {$[4]$} \\
\hline $\mathrm{W}$ & 70 & -0.56 & 0.12 & {$[4]$} \\
\hline $\mathrm{Au}$ & 43 & -0.789 & 0.13 & {$[4]$} \\
\hline $\mathrm{Ag}$ & 39 & -1.063 & 0.257 & {$[4]$} \\
\hline $\mathrm{Zr}$ & 40 & -0.57 & 0.263 & This work \\
\hline $\mathrm{Ti}$ & 40 & -0.222 & 0.234 & This work \\
\hline $\mathrm{Al}$ & 27 & -0.453 & 0.481 & This work \\
\hline
\end{tabular}

\section{RESULTS AND DISCUSSIONS}

\subsection{Nuclear data processing code NECP-Atlas}

NECP-Atlas [11] is a nuclear data processing code developed in Xi'an Jiaotong University in China. The motivation for the development is to establish a platform to carry out deeper researches on nuclear data 
processing method to satisfy the demands on accurate cross sections in the fields of high-fidelity transport simulation and advanced reactor design. Through long-term development and full verification, NECPAtlas is competent to provide cross sections for deterministic and Monte Carlo transport calculation. In order to demand heating and damage calculation, a new module named Heat calc is developed in NECPAtlas, which is used to generate pointwise heat production cross sections and radiation damage energy production for specified reactions and adds them to an existing PENDF file. Damage to materials caused by neutron irradiation is an important design consideration in fission reactors and is expected to be an even more important problem in fusion power systems. In the nuclear data processing code NJOY, the NRT-DPA model is used in HEATR module. In order to obtain more reasonable and accurate radiation damage energy production cross section, both NRT-DPA model and ARC-DPA model are used in Heat_calc module of NECP-Atlas.

\subsection{Number of defects atoms comparison}

In order to verify the accuracy of the fitting material constants in this paper, the number of defects atoms calculated by ARC-DPA model using the fitting material constants in this paper is compared with the results of MD simulations. As shown in Fig. 2, the results in this paper using ARC-DPA model agree well with the results of MD simulations.

It can be got from Fig. 2 that the steady vacancies predicted by the NRT-DPA model is greater than that of the MD simulations. This can be explained by the fact that the NRT model does not accurately describe the atomic interactions at the thermal spike. Since the defect center at the thermal spike has a significant kinetic energy, it contributes to the vacancy recovery, and then results in a significant reduction in the number of vacancies. In order to observe the difference beween NRT-DPA model and ARC-DPA model, the efficiency functions are ploted in Fig. 3. The efficiency is also the fraction of remaining vacancies. As shown in Fig. 3, the fraction of remaining vacancies equal to 1 when the damage energy is less than $2 E_{d} / 0.8$. In addition, the efficiency is decresed with damage energy and has a minimum value $\mathrm{c}$ when the damage energy is infinite.

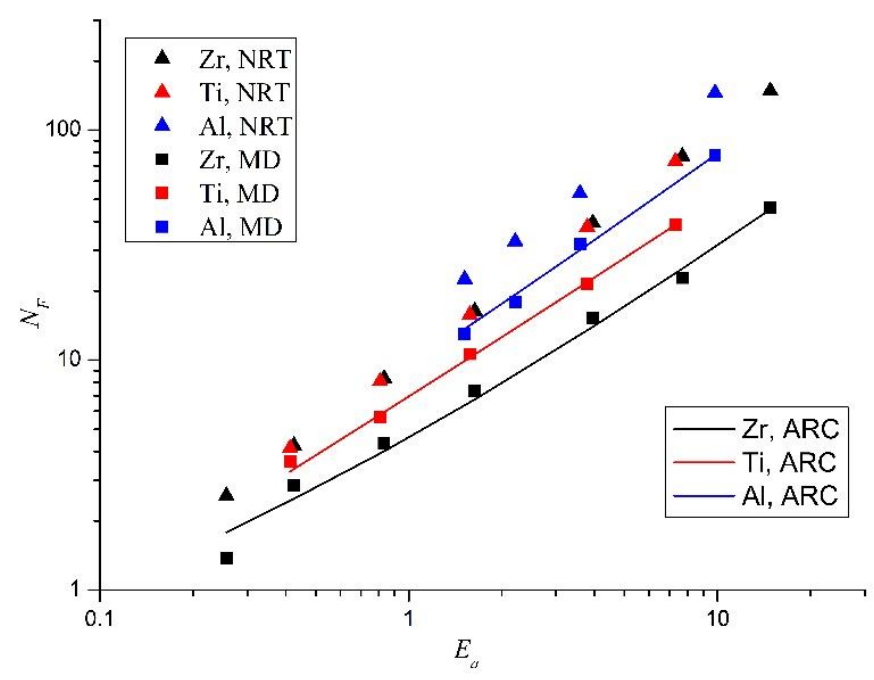

Figure 2. Number of defects atoms comparison

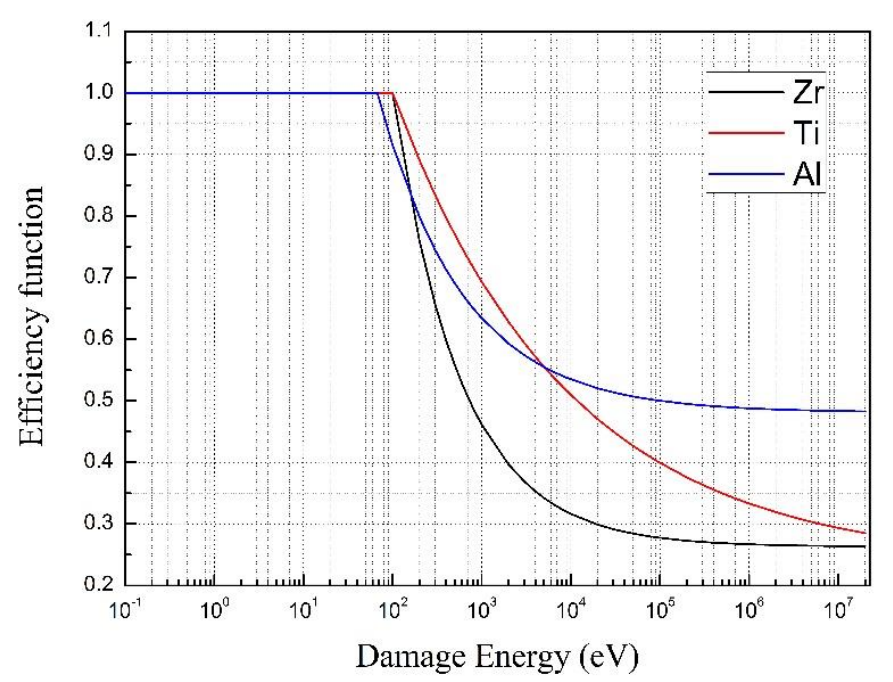

Figure 3. Efficiency function for $\mathrm{Zr}$, Ti and Al 


\subsection{Comparison of radiation damage energy production cross section}

As mentioned in many literatures [12-13], a bug that 1 DPA for damage energy between $E_{d}$ and $2 E_{d} / 0.8$ is missing appear in the NJOY code. In Ref. 12, the code corrections are proposed. But a mistake that the corrected code has thought of the PKA energy as the damage energy is made. In order to verify the accuracy of Heat_calc module in NECP-Atlas, the bugs of NJOY2016 code [14] are modified to make it as the reference.

Recently, a DPA cross sections database based on both NRT-DPA model and ARC-DPA (or a combined MD-BCA) model have been added to the JEFF-3.3 nuclear data library [15]. After fully validated, it is found that the DPA cross sections database given in JEFF-3.3 nuclear data library gives error data because the DPA cross sections database is generated by NJOY using wrong corrected code proposed in Ref. 12.

In order to verify the accuracy of the radiation damage energy production cross section produced by NECP-Atlas, the radiation damage energy production cross sections are compared with that generated by NJOY2016 using modified code. The evaluated nuclear database used in this paper is ENDF/B-VIII [16]. As shown in Fig. 4 and Fig. 5, the radiation damage energy production cross sections of Fe-56 and Zr-90 by NRT-DPA model and their respective bias compared with NJOY2016 are given. At the same time, the radiation damage energy production cross sections by ARC-DPA model are also given in Fig. 4 and Fig. 5. It can be seen from Fig. 4 and Fig. 5 that the bias of radiation damage energy production cross sections of Fe-56 and Zr-90 between NECP-Atlas using NRT-DPA model and NJOY2016 is very small. Moreover, it can be concluded that the radiation damage energy production cross sections using ARCDPA model are much smaller than that using NRT-DPA model.

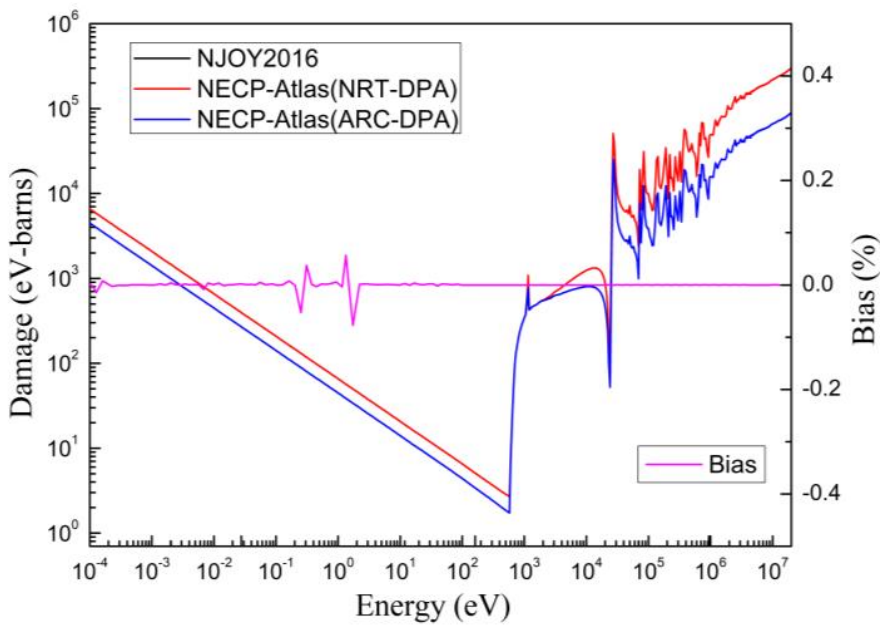

Figure 4. Radiation damage energy production cross sections of Fe-56

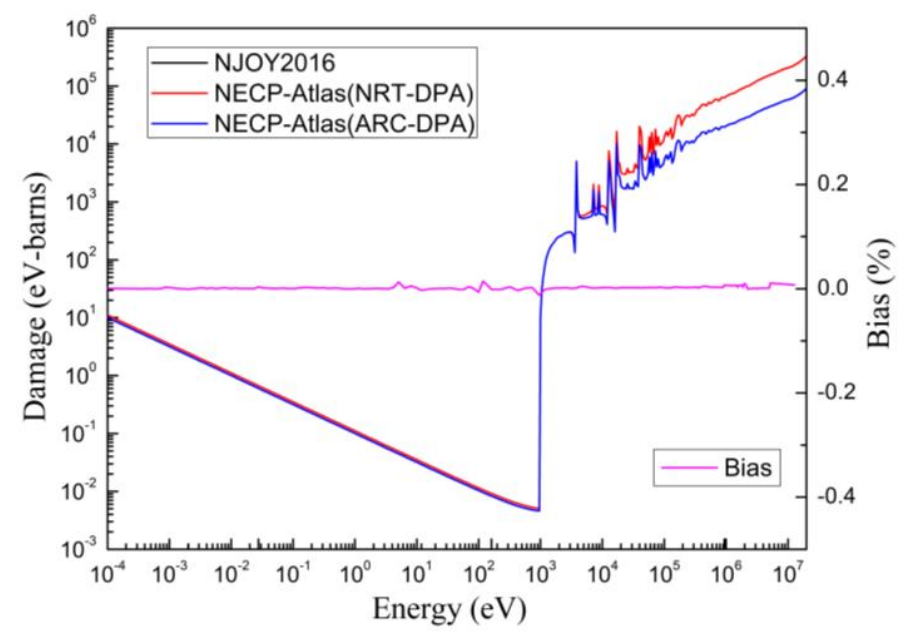

Figure 5. Radiation damage energy production cross sections of $\mathrm{Zr}-90$

\subsection{DPA calculation}

The spectrum of D-T (ITER) is plotted in Fig. 6. The calculated values of DPA in Fe-56 and Zr-90 for 1 full power year (EFPY) of operation in D-T (ITER) are presented in Table 2. It can be got that the calculated values of DPA using ARC-DPA model is only $\sim 1 / 3$ that using NRT-DPA model. It is worth 
noting that with the development of state-of-the-art model, the designer should simply re-adjust the scale which correlates the measured material property with the newly estimated DPA as mentioned in Ref. 17.

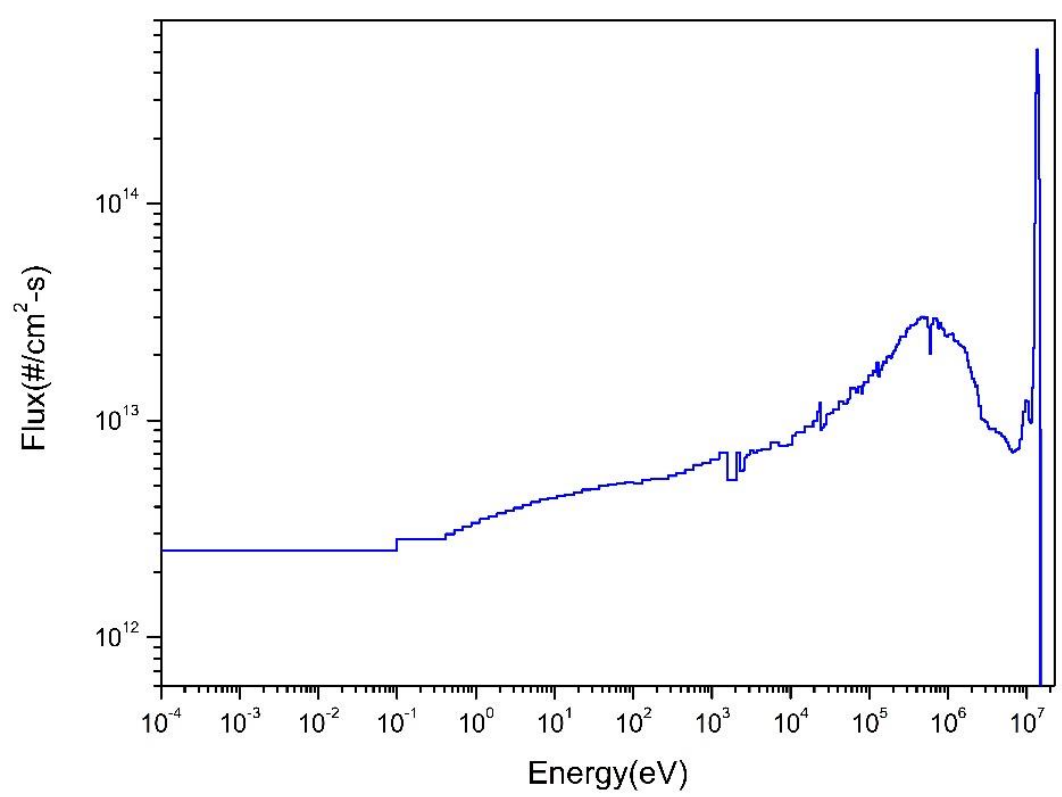

Figure 6. Neutron spectra of D-T (ITER)

Table II. Calculated values of DPA

\begin{tabular}{|c|c|c|}
\hline Material & NRT-DPA & ARC-DPA \\
\hline $\mathrm{Fe}$ & 4.91 & 1.51 \\
\hline $\mathrm{Cu}$ & 4.99 & 1.46 \\
\hline
\end{tabular}

\section{CONCLUSIONS}

DPA is a key factor for material of nuclear systems exposing to high levels of incident radiations. The DPA value is related to the physical and mechanical properties of material of nuclear systems. It is an important work to evaluate the DPA of material. Radiation damage energy production cross section generated by nuclear data processing code is an important data used to calculate DPA. A state-of-the-art model named ARC-DPA model which can provide more physically realistic descriptions of primary defect creation in materials is used in the nuclear data processing code NECP-Atlas. Moreover, material constants of some materials are given in this paper firstly. Through comparing with the results of MD simulations, it can be concluded that the results using material constants fitted in this paper have very high accuracy. Numerical results show that NECP-Atlas adopting NRT-DPA model can give the same accuracy as NJOY2016. Further, NECP-Atlas can also give radiation damage energy production cross sections using the ARC-DPA model. The DPA value using the ARC-DPA model is only $\sim 1 / 3$ of that using the NRT-DPA model. 


\section{ACKNOWLEDGMENTS}

This research was supported by the National Key R\&D Program of China (No. 2017YFE0302200).

\section{REFERENCES}

1. G.H. Kinchin and R.S. Pease, "The displacement of atoms in solids by radiation," Rep. Prog. Phys. 18(1), pp. 77-86 (1955).

2. M.J. Norgett, M.T. Robinson and I.M. Torrens, "A proposed method of calculating displacement dose rates," Nucl. Eng. Des. 33(1), pp. 50-54 (1975).

3. S.J. Zinkle and B.N. Singh, "Analysis of displacement damage and defect production under cascade damage conditions," J. Nucl. Mater. 199, pp. 173-191 (1993).

4. K. Nordlund, S.J. Zinkle et al., "Improving atomic displacement and replacement calculations with physically realistic damage models," Nat. Commun. 9(1), pp. 173-191 (2018).

5. A.Y. Konobeyev, U. Fischer et al., "Evaluation of effective threshold displacement energies and other data required for the calculation of advanced atomic displacement cross-sections," Nucl. Eng. Technol. 3(3), pp. 169-175 (2017).

6. J. Lindhard, V. Nielsen et al., "Integral equations governing radiation effects," Mat. Fys. Medd. Dan. Vid. Selsk. 33(10), pp. 1-42 (1963).

7. M.T. Robinson and I.M. Torrens, "Computer simulation of atomic-displacement cascades in solids in the binary-collision approximation," Phys. Rev. B 9(12), pp. 5008-5024 (1974).

8. M.T. Robinson, "Energy dependence of neutron radiation damage in solids," Oak Ridge National Lab., Tenn. (1970).

9. D.J. Bacon, F. Gao et al., "The primary damage state in fcc, bcc and hcp metals as seen in molecular dynamics simulations," J. Nucl. Mater. 276, pp. 1-12 (2000).

10. S.J. Wooding, L.M. Howe et al., "A molecular dynamics study of high-energy displacement cascades in a-zirconium," J. Nucl. Mater. 254, pp. 191-204 (1998).

11. T. Zu, J. Xu et al., "NECP-Atlas: A new nuclear data processing code," Ann. Nucl. Eng. 123, pp. 153161 (2019).

12. "Comparison of NRT-dpa cross sections calculated by NJOY-2012.50 with ASTM standard for ${ }^{\text {nat } F e, " ~}$ https://www-nds.iaea.org/CRPdpa/NJOY-dpa_vs_ASTM693.pdf (2015).

13. S. Chen, D. Bernard and L. Buiron, "Study on the self-shielding and temperature influences on the neutron irradiation damage calculations in reactors," Nucl. Eng. Des. 346, pp. 85-96 (2019).

14. J.L. Conlin et al., "OpenSource Release of NJOY2016 and NJOY21," https://njoy.github.io/NJOY2016.

15. "The Joint Evaluated Fission and Fusion File, JEFF-3.3," https:/www.oecd-nea.org/dbdata/jeff/.

16. D.A. Brown et al., "ENDF/B-VIII.0: The $8^{\text {th }}$ major release of the nuclear reaction data library with CIELO-project cross sections, new standards and thermal scattering data," Nucl. Data. Sheets. 148, pp. 1-142 (2018).

17. U. Saha et al., "Application of arc-dpa model to estimate the primary radiation damage of structural materials by neutrons and the necessity of rescaling dpa versus final experimental damage correlations," J. Nucl. Mater. 522, pp. 86-96 (2019). 